\title{
La Responsabilidad Social en la Uladech Católica en el contexto de la reforma universitaria
}

\author{
Julio B. Domínguez Granda*
}

Hélmer T. Chávez Pérez

Perú

\section{Resumen}

El presente trabajo se hace con el propósito de dar a conocer cómo la Universidad Católica Los Ángeles de Chimbote ha logrado institucionalizar la Responsabilidad Social como un eje transversal en la formación integral de los estudiantes, como parte de la transformación universitaria producto de la nueva Ley Universitaria 30220.

\section{Introducción}

La universidad tiene un lugar especial en la sociedad y desempeña un papel irreemplazable en ella; un lugar no solo físico, sino

* Doctor en Ciencias Políticas y Relaciones internacionales, rector de la Universidad Católica Los Ángeles de Chimbote. Contacto: rectorado@uladech. edu.pe

* Doctor en Educación, director de Responsabilidad Social de la Universidad Católica Los Ángeles de Chimbote. Contacto: dires@uladech.edu.pe 
fundamentalmente cultural, social, de promoción de la investigación, del desarrollo (Martínez, 2007).

La Universidad tiene múltiples desafíos en este siglo XXI y su relación con la sociedad civil es uno de los más importantes, sobre todo cuando desde determinados sectores neoconservadores se enfatiza que esa vinculación debe estar más cercana al mundo laboral y empresarial que a las necesidades sociales emergentes, todo ello en un contexto de desigualdades donde la función de responsabilidad pública debe apostar decididamente por fomentar políticas de redistribución y reconocimiento que promuevan la inclusión y la participación democrática desde las diferentes áreas de conocimiento (Vila, 2012).

El término Responsabilidad Social comenzó a utilizarse de forma generalizada a comienzos de la década de 1970, aunque organizaciones y gobiernos ya realizaban acciones en algunos de los aspectos considerados como responsabilidad social desde finales del siglo XIX, y en algunos casos, incluso desde antes.

En el pasado, la atención sobre responsabilidad social se había centrado principalmente en las empresas. Para la mayoría de las personas el término "responsabilidad social corporativa" es todavía más familiar que el de "responsabilidad social".

La visión de que la responsabilidad social es aplicable a todas las organizaciones surgió en la medida en que diferentes tipos de organizaciones, no solo aquellas del mundo de los negocios, reconocieron que ellas también tenían responsabilidades en la contribución al desarrollo sostenible (Indecopi, 2010).

A nivel académico, principalmente en la educación superior, progresivamente se han venido incorporando diferentes materias a la responsabilidad social; hace más de un siglo aparecen las prácticas laborales y las prácticas justas de operación, actualmente, los derechos humanos, el medio ambiente, la protección del consumidor y la lucha contra el fraude y así otros que llegan a verse como elementos importantes de la responsabilidad social, sin descartar por ello el tema de la filantropía (Domínguez, 2015).

La Responsabilidad Social puede ofrecer numerosos beneficios potenciales para una organización como sigue: toma de decisiones con base en la compresión de las expectativas de la sociedad y el riesgo de 
ignorarla, gestión de riesgos, gestión de la innovación, mejora competitiva, mejora de su relación con las partes interesadas, mejora del clima organizacional, mejora de la salud y seguridad en el trabajo, mejora de la motivación y retención del personal, ahorros por mejora de productividad, entre otros (Domínguez, 2015).

El interés creciente hacia los aspectos de responsabilidad social y ética de las universidades es una respuesta a la llamada "sed de eticidad”, que señaló con gran acierto F. Vallaeys, ante los grandes problemas sociales de inequidad, corrupción, escándalos financieros, ausencia de valores morales, falta de justicia social, entre otros. Esta problemática ha promovido la reflexión sobre la calidad de la formación de los profesionales y el papel de la universidad en la sociedad (Ojalvo y Gonzáles, 2014).

La Universidad puede programar, patrocinar, fomentar, articular y/o crear instancias en función de la Responsabilidad Social Universitaria, como también reconocer experiencias ya existentes que representan este espíritu. En este sentido, se entrega una muestra de iniciativas que se explicitan a continuación, enmarcadas en 4 tipos de experiencias: Gestión-Acceso y Permanencia-Docencia Vinculación (Figueroa, 2014).

Otras alternativas para enfocar la responsabilidad social son las prácticas profesionales y pasantías, las cuales están en la mira de las reformas curriculares llevadas a cabo bajo la modalidad del currículo integral basado en competencias, con la finalidad de garantizar la inserción de los estudiantes y docentes en la problemática social (Salom, 2011).

En Perú, de acuerdo a la nueva Ley Universitaria N.³0220, art. $124^{\circ}$, la responsabilidad social universitaria es la gestión ética y eficaz del impacto generado por la universidad en la sociedad debido al ejercicio de sus funciones: académicas, de investigación y de servicios de extensión y participación en el desarrollo nacional en sus diferentes niveles y dimensiones; incluye la gestión de impactos producido por las relaciones entre los miembros de la comunidad universitaria, sobre el ambiente, y sobre las organizaciones públicas y privadas que se constituyen en partes interesadas. Así mismo, entendemos que la responsabilidad social universitaria es fundamento de la vida universitaria, contribuye al desarrollo sostenible y al bienestar de la sociedad, pero sobre todo compromete a toda la comunidad universitaria. 
La política de responsabilidad social en la Uladech Católica se incluye en los diversos documentos estratégicos, como son: Proyecto Educativo Institucional, Plan Estratégico Institucional 2016-2018, Plan de Funcionamiento y Desarrollo Institucional 2016; además en los documentos normativos como son: Estatuto, Reglamento General, Reglamento de Organización y Funciones por Procesos, Manual de Organización y Funciones, Reglamento Académico, Reglamento de Extensión Cultural y Proyección Social y Reglamento de Servicio Social Universitario (Uladech Católica, 2016).

\section{Antecedentes de la RS en Uladech Católica}

El resultado de la transformación de la Uladech Católica para incluir el modelo de gestión de la responsabilidad social se inicia a partir de1997, año en el cual se implementa un modelo humanista cristiano basado en la ética y valores incluyendo asignaturas obligatorias (Pastoral Universitaria, Ética y Valores) en el plan de estudio de todas las carreras profesionales. En el año 2006 se crea Departamento Académico de Responsabilidad Social Universitario acorde a la concepción moderna de Ética y Valores que relaciona la Responsabilidad Social Universitaria en un mundo globalizado. A partir del año 2008, se realizó la implementación del Modelo del Sistema de Responsabilidad Social, integrado por nueve asignaturas, que incluye la estrategia pedagógica del desarrollo de proyectos de extensión universitaria y proyección social alineados a la Iso 26000. Posteriormente, como consecuencia de las mejoras organizacionales generadas por el proceso de acreditación de las carreras profesionales, se implementó en la Universidad la gestión por procesos, erigiéndose la Dirección de Responsabilidad Social. Estas mejoras determinaron en los proyectos educativos de las escuelas profesionales el cambio en la denominación de las asignaturas por ocho asignaturas de Responsabilidad Social (Responsabilidad Social I-VIII).

En 2014 se publica en el Diario Oficial El Peruano (2014) la Ley Universitaria N. ${ }^{\circ} 30220$, que en sus artículos 124 y 125 precia la definición de la responsabilidad social universitaria y los medios de promoción de 
esta, las que han sido adecuadas en el Estatuto de la Universidad. En el artículo 130 de la indicada Ley se establece la creación del servicio social universitario, que consiste en la realización obligatoria de actividades temporales que ejecuten los estudiantes universitarios de manera descentralizada; tendientes a la aplicación de los conocimientos que hayan obtenido y que impliquen una contribución en la ejecución de las políticas públicas de interés social y fomenten un comportamiento altruista y solidario que aporte en la mejora de la calidad de vida de los grupos vulnerables en nuestra sociedad. Como se puede observar, este modelo ya venía siendo ejecutado por la universidad.

Dentro de las acciones de adecuación a la nueva Ley Universitaria en su artículo 130 se aprobó el Reglamento del Servicio Social Universitario (Uladech, 2015) y, posteriormente se elaboró el Programa del Servicio Social Universitario (ssu) que se inició en el Semestre 2015-1 con la asignatura de RS VII, continuando en el 2015-2 con la asignatura de RS VIII, según lo reglamentado; implementándose proyectos orientados a la prestación del servicio social universitario según la actividad clave de la carrera profesional.

$\mathrm{Al}$ respecto cabe establecer la pregunta eje de esta investigación: ¿cuál es el impacto que ha tenido la institucionalización de la Responsabilidad Social como un eje transversal en la formación integral de los estudiantes, la Universidad Católica Los Ángeles de Chimbote, en el marco de Ley Universitaria 30220? Tema que se pretende analizar con el propósito de caracterizar la prácticas en la formación integral de los estudiantes, en la Universidad Católica Los Ángeles de Chimbote, en el marco de Ley Universitaria 30220.

\section{Metodología}

\section{Fuentes de información}

El módulo de proyectos proporciona la información relacionada con proyectos presentados y los proyectos que fueron culminados. Así mismo, el ERP University cuenta con el módulo de encuestas que ha permitido procesar la información proveniente de los cuestionarios elaborados especialmente para el caso y aplicados a los docentes, estudiantes y 
poblaciones vulnerables para el cálculo de grado de satisfacción de las partes interesadas.

\section{Cálculo de la eficacia en el desarrollo de proyectos del servicio social universitario}

Estudiantes y docentes tutores de RS registran los proyectos que desarrollan en poblaciones vulnerables de los cinturones de pobreza de regiones del país, en donde está presente la Sede Central y filiales de Uladech Católica. Según dicho registro, cuyos reportes permiten calcular la eficacia de desarrollo de proyecto como porcentaje de medición de eficacia del 2015, utilizando la siguiente relación:

$\left(\mathrm{N} .^{\circ}\right.$ de informes finales aprobados de proyectos ejecutados $/ n^{\circ}$ de proyectos aprobados) $\mathrm{x} 100$

\section{Cálculo de la satisfacción y otras características en el desarrollo de proyectos del servicio social universitario}

Con los datos proporcionados por la aplicación online de la encuesta de satisfacción a docentes, estudiantes y grupos vulnerables provenientes del módulo de encuestas se procede a establecer los porcentajes de satisfacción de cada grupo.

Las encuestas de estudiantes consideran lo siguiente: a) frecuencia en que el grupo de interés colaboró con el servicio prestado, b) adquisición de conocimientos vinculados con su carrera profesional, c) respeto, amabilidad y tolerancia hacia los beneficiarios, d) contribución para mejorar las condiciones de vida de la comunidad, e) satisfacción con el servicio social, f) satisfacción con las experiencias obtenidas en la ejecución del servicio social.

Las encuestas a los docentes consideran lo siguiente: a) importancia del servicio social, b) verificación de la vulnerabilidad de las poblaciones elegidas, c) articulación de asignaturas de responsabilidad social con los proyectos de servicio social, d) verificación del aporte del servicio social a las mejoras de las condiciones de vida de las comunidades vulnerables, e) verificación del conocimiento del docente 
respecto a la normativa de responsabilidad social universitaria y del servicio social, f) interés de los estudiantes por el desarrollo del servicio social, g) opción por la mejora del servicio social.

Las encuestas a los grupos de interés consideran lo siguiente: a) mejora de calidad de vida, b) ayuda oportuna y solidaria a la población por los estudiantes, c) satisfacción con el servicio prestado por los estudiantes, d) mantenimiento del servicio de los estudiantes, e) satisfacción con el servicio social.

\section{Resultados y discusión}

\section{Eficacia del servicio social}

En el cuadro1 se presenta el acumulado de proyectos ssu aprobados e informes finales evidenciados por los estudiantes en cada uno de los semestres considerados en el primer grupo de ssu correspondiente al semestre 2015-1 y 2015-02, así mismo el total acumulado en el periodo.

Con la información del cuadro2, se satisface la relación para obtener el índice de eficacia de los proyectos de servicio social como porcentaje de proyectos con informe final para 2015 alcanzado por los estudiantes: porcentaje de eficacia del ssu $=(2722 / 2769) \times 100 ;=98,3 \%$

De acuerdo con lo anterior, los estudiantes de la Uladech Católica muestran una eficacia del $98,3 \%$ de proyectos con informe final respecto a proyectos presentados para ejecución.

Cuadro 1. Proyectos Ssu presentados y aprobados. Estudiantes, 2015

\begin{tabular}{|c|c|}
\hline $2015-1$ & Proyectos \\
\hline RS VII - Proyectos aprobados & 1587 \\
\hline RS VIII - Proyectos aprobados & 1182 \\
\hline Total & 2769 \\
\hline RS VIII -Informes finales aprobados & 912 \\
\hline $2015-2$ & \\
\hline RS VIII - Informes finales aprobados & 1810 \\
\hline 2015 & \\
\hline Total de informes finales (Sumatoria de semestre 2015-1y 2015-2) & 2722 \\
\hline
\end{tabular}

Fuente: módulo PECPS-Ssu, 2015. 


\section{Satisfacción y otros impactos del ssu}

El grado de satisfacción del ssu se aplica para docentes, estudiantes y grupos vulnerables a través de encuestas cuyos resultados se presentan en los cuadros N. ${ }^{\circ} 2,3,4,5,6$ y 7 ; para estudiantes, N. $.^{\circ} 8,9,10$, $11,12,13$ y 14 ; para docentes y $\mathrm{N}^{\circ} 15,16,17,18$ y 19 para grupos de interés, de acuerdo a los aspectos considerados en la metodología. En lo que sigue se discutirán independientemente los casos de estudiantes, docentes y grupos de interés de acuerdo a los cuadros de resultados correspondientes:

\section{Estudiantes}

En lo que sigue se presentan los resultados de las encuestas aplicadas a los estudiantes que muestran el grado de satisfacción y otros aspectos positivos relacionados con el servicio social tal como se describe en los siguientes cuadros, y en cuanto respecta a la satisfacción con las experiencias obtenidas en la ejecución del servicio social, los resultados han sido interpretados al final de la presentación de los cuadros.

Cuadro 2. Colaboración e interés de la comunidad en el ssu

\begin{tabular}{|l|c|c|}
\hline \multicolumn{1}{|c|}{ Opción } & Frecuencia & Porcentaje \% \\
\hline Nunca & 27 & 2 \\
\hline A veces & 247 & 20 \\
\hline Siempre & 967 & 78 \\
\hline Total & 1241 & 100 \\
\hline
\end{tabular}

Fuente: elaboración propia.

Cuadro 3. Utilidad de los conocimientos adquiridos durante su formación profesional en situaciones prácticas para solucionar problemas de la comunidad

\begin{tabular}{|l|c|c|}
\hline \multicolumn{1}{|c|}{ Opción } & Frecuencia & Porcentaje \% \\
\hline No & 13 & 1 \\
\hline Sí & 1228 & 99 \\
\hline Total & 1241 & 100 \\
\hline
\end{tabular}

Fuente: elaboración propia. 
Cuadro 4. Interacción con la comunidad recibió un trato de respeto, amabilidad y tolerancia

\begin{tabular}{|l|c|c|}
\hline \multicolumn{1}{|c|}{ Opción } & Frecuencia & Porcentaje \% \\
\hline Nunca & 3 & 0 \\
\hline A veces & 102 & 8 \\
\hline Siempre & 1136 & 92 \\
\hline Total & 1241 & 100 \\
\hline
\end{tabular}

Fuente: elaboración propia.

Cuadro 5. Considera que el ssu realizado ha contribuido a mejorar las condiciones de vida de la comunidad

\begin{tabular}{|l|c|c|}
\hline \multicolumn{1}{|c|}{ Opción } & Frecuencia & Porcentaje \% \\
\hline No & 21 & 2 \\
\hline Sí & 1220 & 98 \\
\hline Total & 1241 & 100 \\
\hline
\end{tabular}

Fuente: elaboración propia.

Cuadro 6. Percepción en relación si la comunidad quedó satisfecha con el ssu

\begin{tabular}{|l|c|c|}
\hline \multicolumn{1}{|c|}{ Opción } & Frecuencia & Porcentaje \% \\
\hline No & 29 & 2 \\
\hline Sí & 1212 & 98 \\
\hline Total & 1241 & 100 \\
\hline
\end{tabular}

Fuente: elaboración propia.

Cuadro 7. Satisfacción con las experiencias obtenidas en la ejecución del ssu

\begin{tabular}{|l|c|c|}
\hline \multicolumn{1}{|c|}{ Opción } & Frecuencia & Porcentaje \% \\
\hline No & 41 & 3 \\
\hline Sí & 1200 & 97 \\
\hline Total & 1241 & 100 \\
\hline
\end{tabular}

Fuente: elaboración propia.

Tal como se aprecia en el cuadro 2 el $78 \%$ de los estudiantes afirma que los miembros de los grupos de interés colaboraron y tomaron 
interés en el servicio prestado por estos en las poblaciones vulnerables y el $20 \%$ lo hace esporádicamente.

- De acuerdo al cuadro 3 el 99\% de los estudiantes afirma que los conocimientos adquiridos durante su formación profesional le sirvieron para solucionar problemas de la comunidad.

- De acuerdo al cuadro 4 el $92 \%$ de los estudiantes considera que el trato recibido por los beneficiarios fue de respeto, amabilidad y tolerancia.

- De acuerdo al cuadro 5 el $98 \%$ de los estudiantes considera que el ssu ha contribuido a mejorar las condiciones de vida de la comunidad.

- De acuerdo al cuadro 6 el $98 \%$ de los estudiantes considera que la comunidad quedó satisfecha con el servicio.

- De acuerdo al cuadro 7 el 97\% de los estudiantes está satisfecho con las experiencias obtenidas en la ejecución del servicio.

\section{Docentes}

En lo que sigue se presentan los resultados de las encuestas aplicadas a los docentes que muestran el grado de satisfacción y otros aspectos positivos relacionados con el servicio respecto a su aporte en la mejora del proceso del servicio social, los cuales han sido interpretados al final de la presentación de los siguientes cuadros:

Cuadro 8. Importancia del ssu

\begin{tabular}{|l|c|c|}
\hline \multicolumn{1}{|c|}{ Opción } & Frecuencia & Porcentaje \% \\
\hline No & 0 & 0 \\
\hline Sí & 47 & 100 \\
\hline Total & 47 & 100 \\
\hline
\end{tabular}

Fuente: elaboración propia. 
Cuadro 9. Considera que las poblaciones elegidas son vulnerables

\begin{tabular}{|l|c|c|}
\hline \multicolumn{1}{|c|}{ Opción } & Frecuencia & Porcentaje \% \\
\hline No & 0 & 0 \\
\hline Sí & 47 & 100 \\
\hline Total & 47 & 100 \\
\hline
\end{tabular}

Fuente: elaboración propia.

Cuadro 10. El ssu se articula como responsabilidad social

\begin{tabular}{|l|c|c|}
\hline \multicolumn{1}{|c|}{ Opción } & Frecuencia & Porcentaje \% \\
\hline No & 0 & 0 \\
\hline Sí & 47 & 100 \\
\hline Total & 47 & 100 \\
\hline
\end{tabular}

Fuente: elaboración propia.

Cuadro 11. El ssu ha contribuido a mejorar las condiciones de vida de las comunidades vulnerables

\begin{tabular}{|l|c|c|}
\hline \multicolumn{1}{|c|}{ Opción } & Frecuencia & Porcentaje \% \\
\hline No & 4 & 9 \\
\hline Sí & 43 & 91 \\
\hline Total & 47 & 100 \\
\hline
\end{tabular}

Fuente: elaboración propia.

Cuadro 12. Conocimiento de normas del ssu

\begin{tabular}{|l|c|c|}
\hline \multicolumn{1}{|c|}{ Opción } & Frecuencia & Porcentaje \% \\
\hline No & 8 & 17 \\
\hline Sí & 39 & 83 \\
\hline Total & 47 & 100 \\
\hline
\end{tabular}

Fuente: elaboración propia.

Cuadro 13. Interés de los estudiantes por el ssu

\begin{tabular}{|l|c|c|}
\hline \multicolumn{1}{|c|}{ Opción } & Frecuencia & Porcentaje \% \\
\hline No & 9 & 19 \\
\hline Sí & 38 & 81 \\
\hline Total & 47 & 100 \\
\hline
\end{tabular}

Fuente: elaboración propia. 
Cuadro 14. Aporte en la mejora del proceso del ssu

\begin{tabular}{|l|c|c|}
\hline \multicolumn{1}{|c|}{ Opción } & Frecuencia & Porcentaje \% \\
\hline No & 0 & 0 \\
\hline Sí & 47 & 100 \\
\hline Total & 47 & 100 \\
\hline
\end{tabular}

Fuente: elaboración propia.

Tal como se aprecia en el cuadro 8 el $100 \%$ de los docentes considera importante el servicio social.

- De acuerdo al cuadro 9 el $100 \%$ de los docentes considera que las poblaciones elegidas son vulnerables.

- De acuerdo al cuadro 10 el $100 \%$ de los docentes considera que el servicio social universitario se articula como responsabilidad social.

- De acuerdo al cuadro 11 el 91\% de los docentes considera que el servicio ha contribuido a mejorar las condiciones de vida de las poblaciones vulnerables.

- De acuerdo al cuadro 12 el $83 \%$ de los docentes conoce las normas del servicio social universitario.

- De acuerdo al cuadro 13 el $81 \%$ de los docentes considera que los estudiantes muestran interés por el servicio social universitario.

- De acuerdo al cuadro 14 el 100\% de los docentes considera que aporta en la mejora del servicio social universitario.

\section{Grupos de interés}

En lo que sigue se presentan los resultados de las encuestas aplicadas a los grupos de interés que muestran el grado de satisfacción y otros aspectos positivos relacionados con el servicio social universitario respecto a la satisfacción con el servicio prestado por los estudiantes, los cuales han sido interpretados al final de la presentación de los cuadros que se presentan a continuación. 
Cuadro 15. El ssu contribuye a mejorar las condiciones de vida de los miembros de la comunidad

\begin{tabular}{|l|c|c|}
\hline \multicolumn{1}{|c|}{ Opción } & Frecuencia & Porcentaje \% \\
\hline No & 0 & 0 \\
\hline Sí & 6 & 100 \\
\hline Total & 6 & 100 \\
\hline
\end{tabular}

Fuente: elaboración propia.

Cuadro 16. Ayuda oportuna y solidaria por parte de los estudiantes del ssu a los miembros de su comunidad

\begin{tabular}{|l|l|l|}
\hline \multicolumn{1}{|c|}{ Opción } & \multicolumn{1}{c|}{ Frecuencia } & Porcentaje \% \\
\hline No & 0 & 0 \\
\hline Sí & 6 & 100 \\
\hline Total & 6 & 100 \\
\hline
\end{tabular}

Fuente: elaboración propia.

Cuadro 17. Satisfacción de los miembros de su comunidad con el ssu prestado por los estudiantes

\begin{tabular}{|l|c|c|}
\hline \multicolumn{1}{|c|}{ Opción } & Frecuencia & Porcentaje \% \\
\hline No & 0 & 0 \\
\hline Sí & 6 & 100 \\
\hline Total & 6 & 100 \\
\hline
\end{tabular}

Fuente: elaboración propia.

Cuadro 18. Preferencia por la continuidad del ssu en la comunidad por los estudiantes

\begin{tabular}{|l|c|c|}
\hline \multicolumn{1}{|c|}{ Opción } & Frecuencia & Porcentaje \% \\
\hline No & 0 & 0 \\
\hline Sí & 6 & 100 \\
\hline Total & 6 & 100 \\
\hline
\end{tabular}

Fuente: elaboración propia. 
Cuadro 19. Satisfacción con el ssu prestado por los estudiantes

\begin{tabular}{|l|c|c|}
\hline \multicolumn{1}{|c|}{ Opción } & Frecuencia & Porcentaje \% \\
\hline No & 0 & 0 \\
\hline Sí & 6 & 100 \\
\hline Total & 6 & 100 \\
\hline
\end{tabular}

Fuente: elaboración propia.

Tal como se aprecia en el cuadro 15 el 100\% de los grupos de interés considera que el servicio social realizado por los estudiantes de Uladech Católica contribuye a mejorar las condiciones de vida de los miembros de la comunidad.

- De acuerdo al cuadro 16 el 100\% de los grupos de interés considera que ha recibido ayuda oportuna y solidaria por parte de los estudiantes del servicio social a los miembros de su comunidad.

- De acuerdo al cuadro 17, el 100\% de los grupos de interés considera que los miembros de su comunidad está satisfecho con el servicio social prestado.

- De acuerdo al cuadro 18 el $100 \%$ de los grupos de interés tiene preferencia por la continuidad del servicio social en la comunidad.

- De acuerdo al cuadro 19 el 100\% de los grupos de interés está satisfecho con el servicio social prestado por los estudiantes

\section{Conclusiones}

Uladech Católica ha demostrado una rápida capacidad de adaptación a los cambios verificando su estrategia de políticas universitarias que siguen las tendencias mundiales, tal es el caso de la responsabilidad social.

El impacto del servicio social universitario en el estudiante universitario ha sido positivo en cuanto a que mayoritariamente los estudiantes que participaron en el programa están de acuerdo en que los miembros de los grupos de interés colaboraron y tomaron interés en el servicio prestado por estos en las poblaciones vulnerables; los conocimientos adquiridos durante su formación profesional le sirvieron 
para solucionar problemas de la comunidad; el trato recibido por los beneficiarios fue de respeto, amabilidad y tolerancia; el servicio social universitario ha contribuido a mejorar las condiciones de vida de la comunidad; la comunidad quedó satisfecha con el servicio social universitario y los estudiantes se encuentran satisfechos con las experiencias obtenidas en la ejecución del servicio social universitario.

El impacto del servicio social universitario en el docente universitario ha sido positivo en cuanto a que en su mayoría los docentes que participaron del programa están de acuerdo en que: el servicio social universitario es importante; las poblaciones elegidas son vulnerables; se articula como responsabilidad social; ha contribuido a mejorar las condiciones de vida de las poblaciones vulnerables; los docentes conocen las normas; los estudiantes muestran interés por el servicio social universitario y los docentes aportan en la mejora del servicio social universitario.

El impacto del servicio social universitario en los grupos de interés ha sido positivo en cuanto a que en su mayoría los grupos de interés que participaron del programa están de acuerdo en que: el servicio social realizado por los estudiantes contribuye a mejorar las condiciones de vida de los miembros de la comunidad; ha recibido ayuda oportuna y solidaria por parte de los estudiantes; los miembros de su comunidad están satisfechos con el servicio social prestado por los estudiantes; preferencia por la continuidad en la comunidad de los estudiantes. 


\section{Referencias}

Diario Oficial El Peruano (2014). Ley Universitaria 30220. Recuperado de https://www.sunedu.gob.pe/files/normatividad/LEY_UNIVERSITARIA.PDF

Domínguez, J. (2015). Responsabilidad Social de las Organizaciones (RSO): Avances y propuestas en América Latina. Fuentes de la Responsabilidad Social. Trujillo, Perú: Gráfica Real s.A.c.

Figueroa, L. (2014). La responsabilidad social universitaria en la universidad de Santiago de Chile: algunas iniciativas de nuestro sello como institución estatal y pública. Santiago de Chile: Editorial Universidad de Santiago de Chile.

Idecopi (2010). Norma Técnica Peruana, Guía de Responsabilidad Social, NTP-Iso 26000. Lima, Perú.

Martínez, M. (2007). Aprendizaje, servicio y responsabilidad social de las universidades. Barcelona: Ediciones Octaedro, S.L.

Ojalvo, V. y Gonzales, B. (2014). La responsabilidad social universitaria para la formación. Cuba: Editorial Universitaria.

Salom, J. D. (2011). Responsabilidad social desde el enfoque de la gestión pública en el sector universitario. Revista de Ciencias Sociales. Zulia.

Uladech Católica (2016). Estatuto Institucional. Obtenido de http://www. uladech.edu.pe/images/stories/universidad/documentos/2016/estatuto_ v013_2016.pdf

Uladech Católica (2016). Manual de Organización y Funciones. Recuperado de http://www.uladech.edu.pe/images/stories/universidad/documentos/2016/manual_organizacion_funciones_v006.pdf

Uladech Católica (2016). Plan de Funcionamiento y Desarrollo Institucional 2016. Recuperado de http://www.uladech.edu.pe/images/stories/universidad/documentos/2016/plan-de-funcionamiento-y-desarrollo-institucional-2016-v001.pdf

Uladech Católica (2016). Plan Estratégico Institucional 2016-2018. Recuperado de http://www.uladech.edu.pe/images/stories/universidad/documentos/2016/plan-estrategico-institucional-2016-2018-v001.pdf

Uladech Católica (2016). Proyecto Educativo Institucional. Recuperado de http://www.uladech.edu.pe/images/stories/universidad/documentos/2014/ proyecto-educativo-institucional-v03.pdf 
Uladech Católica (2016). Reglamento Académico, Reglamento de Extensión Cultural y Proyección Social. Recuperado de http://www.uladech.edu. pe/images/stories/universidad/documentos/2017/reglamento-academico-012.pdf

Uladech Católica (2016). Reglamento de Organización y Funciones por Procesos. Recuperado de http://www.uladech.edu.pe/images/stories/universidad/ documentos/2016/reglamento_organizacion_funciones_proceso_v007.pdf

Uladech Católica (2016). Reglamento de Servicio Social Universitario. Recuperado de http://www.uladech.edu.pe/images/stories/universidad/documentos/2016/reglamento_servicio_social_universitario_v03.pdf

Uladech Católica (2016). Reglamento General. Recuperado de http://www. uladech.edu.pe/images/stories/universidad/documentos/2016/reglamento_general_v010.pdf

Vila, E. (2012). Políticas educativas globales y responsabilidad social de las universidades. Málaga: Servicio de Publicaciones y Divulgación Científica de la Universidad de Málaga. 
